\title{
Role of the Medial Preoptic Area in the Neural Control of the Nocturnal Prolactin Surge in the Rat
}

\author{
Jun ARITA AND Masazumi KAWAKAMI
}

\author{
Department of Physiology, Yokohama City University School of \\ Medicine, 2-33 Urafune-cho, Minami-ku, Yokohama
}

\begin{abstract}
In the present study, the role of the medial preoptic area (MPO) in the neural control of the nocturnal prolactin (PRL) surge was investigated in ovariectomized rats. Cervical stimulation (CS) or bilateral MPO lesions caused a marked nocturnal PRL surge at $0400 \mathrm{~h}$ on the fourth day after CS or the lesions in ovariectomized rats in which blood samples were obtained by decapitation. However, operation for indwelling a catheter and serial blood collection completely eliminated the MPO lesioninduced nocturnal surge while they did not affect the CS-induced surge. On the other hand, MPO lesions could not induce the nocturnal PRL surge in neonatally androgenized female rats. These results suggest that the MPO not only tonically inhibits the initiation of the nocturnal PRL surge but also has a buffer action on the PRL surge-suppressing action of stress. Furthermore, it may be possible that the failure of CS to initiate and maintain the nocturnal PRL surge in neonatally androgenized rats is not due to the inability of CS to disinhibit the inhibitory action of the MPO, but rather due to the extinction of the circadian rhythm itself of the nocturnal PRL surge in these rats.
\end{abstract}

Stimulation of the uterine cervix (CS) of the female rat elicits a nocturnal surge of prolactin (PRL) secretion occurring in the early morning and a diurnal surge occurring in the late afternoon (Freeman et al., 1974), regardless of the presence of the ovaries (Smith and Neill, 1976). These two PRL surges have some distinctive features in the neural regulation of their secretion. For example, a variety of noxious procedures such as catheterization, bleeding and sham operations selectively suppresses the diurnal but not nocturnal surge during pseudopregnancy (Freeman et al., 1974).

It has been shown that rats with lesions restricted to the medial preoptic area (MPO) spontaneously exhibit repeated periods of

Received September 2, 1981. pseudopregnancy without CS (Clemens et al., 1976; Brown-Grant et al., 1977; Wiegand et al., 1980). This type of pseudopregnancy seems similar to the CS-induced pseudopregnancy but is characterized by the nocturnal PRL surge without the diurnal surge (Freeman and Banks, 1980).

On the other hand, pseudopregnancy cannot be initiated and maintained in castrated male rats bearing a transplanted ovary and in neonatally androgenized female rats (Zeilmaker, 1963, 1964). It has been shown that the lack of the daily PRL surges is responsible for the sexual differentiation in pseudopregnancy (de Greef $e t$ al., 1980; kawakami and Arita, 1981a). However, the neural mechanism of the lack of the PRL surge in neonatally androgenized rats is not known. 
In the present study, we compared the pattern of the nocturnal PRL surges induced by CS and by MPO lesions in catheterized and decapitated rats to study differential regulation of these nocturnal surges of PRL secretion. We also examined whether or not MPO lesions induce the nocturnal surge in neonatally androgenized female rats to study the involvement of the MPO in the sexual differentiation of the daily PRL surge.

\section{Materials and Methods}

\section{Animals}

Female rats of the Wistar strain, weighing 200 $240 \mathrm{~g}$, were housed under controlled conditions of light (lights on from 0500 to $1900 \mathrm{~h}$ ) and temperature (21-24 ${ }^{\circ} \mathrm{C}$ ) and allowed free access to water and laboratory chew. Ovariectomy was performed under ether anesthesia.

\section{MPO lesions}

Two or three weeks after ovariectomy, the rats received bilateral electrolytic lesions of the MPO or sham lesions. Bilateral lesions of the MPO were placed stereotaxically with a monopolar platinum electrode under ether anesthesia, as described elsewhere (Kawakami et al., 1980). Stereotaxic coordinates for MPO lesions were : anterior, $8.3 \mathrm{~mm}$; vertical, $3.8 \mathrm{~mm}$ above horizontal zero; lateral 0.8 $\mathrm{mm}$, according to the atlas of Albe-Fessard et al. (1966). The lesions were made by applying an anodal direct current of $1.0 \mathrm{~mA}$ through the electrode for $20 \mathrm{sec}$. To sham-lesioned animals, the electrode was inserted but the current was not applied.

At the end of the experiments, the brains were fixed in $10 \%$ formalin, and sectioned at $50 \mu \mathrm{m}$. The sections were stained with cresyl violet and examined with a microscope.

\section{Cervical stimulation}

The uterine cervix of ovariectomized rats was stimulated by a combination of mechanical and electrical stimulation at $1900-2000 \mathrm{~h}$ (first CS) and at $0900-1000 \mathrm{~h}$ on the next day (second CS), as described previously (Kawakami and Arita, 1981a). After mechanical stimulation of $1 \mathrm{~min}$, rectangular pulses of $1 \mathrm{msec}$ duration and $5 \mathrm{~mA}$ amplitude were applied at a frequency of $200 \mathrm{~Hz}$ through an electrode constructed for CS. The pulses were delivered for periods of $10 \mathrm{sec}$ on $/ 5 \mathrm{sec}$ off over a total period of $1 \mathrm{~min}$.
Experiment I Comparison of the nocturnal $P R L$ surges induced by $C S$ and MPO lesions in catheterized and decapitated rats.

Ovariectomized rats with MPO lesions or sham lesions and rats with or without CS were decapitated to obtain blood samples at 0400 and $1200 \mathrm{~h}$ on the fourth day after lesion or first CS. Similarly treated rats were catheterized under ether anesthesia on the day after lesion or first CS, and then the catheter was flushed with saline containing heparin once a day. Serial blood samples $(0.3 \mathrm{~m} l)$ were obtained through the catheter at 1900 and $2300 \mathrm{~h}$ on the third day and at 0400,0600 and $1200 \mathrm{~h}$ on the fourth day.

Experiment II Effect of MPO lesions on PRL secretion in neonatally adrogenized rats

Female pups were given a single subcutaneous injection of $250 \mu \mathrm{g}$ testosterone propionate in 0.05 $\mathrm{m} l$ sesame oil on day 5 of life (the day of birth was designated as day 1). When the body weights of the animals reached approximately $220 \mathrm{~g}$, the ovaries were removed and inspected to check whether or not corpora lutea were present. The ovariectomized rats neonatally treated with testosterone propionate were decapitated at 0400 and $1200 \mathrm{~h}$ on the fourth day after MPO lesions.

\section{PRL measurement and statistical analysis}

The blood was allowed to clot at $4{ }^{\circ} \mathrm{C}$. The serum was separated by centrifugation and stored at -20 ${ }^{\circ} \mathrm{C}$ until assayed. Serum PRL was measured with the radioimmunoassay kit provided by the NIAMDD. Serum PRL concentrations were expressed in terms of NIAMDD-rat-PRL-RP-1. Differences in serum PRL concentrations among groups were analysed by analysis of variance. When the $\mathrm{F}$ ratio was significant, Duncan's multiple range test was used to determine which groups differed.

\section{Results}

\section{Location of MPO lesions}

The lesions, of which the mean diameter was $1.1 \mathrm{~mm}$, damaged most of the dorsal part of the MPO, as shown in Fig. 1, although the ventral parts remained intact in most of the rats. Some of the lesions extended dorsally into a part of the anterior commissure and bed nucleus of the stria terminalis, and rostrally to the posterior border of the diagonal band of Broca. On the other hand, the suprachiasmatic nucleus, medial basal part of the suprachiasmatic 

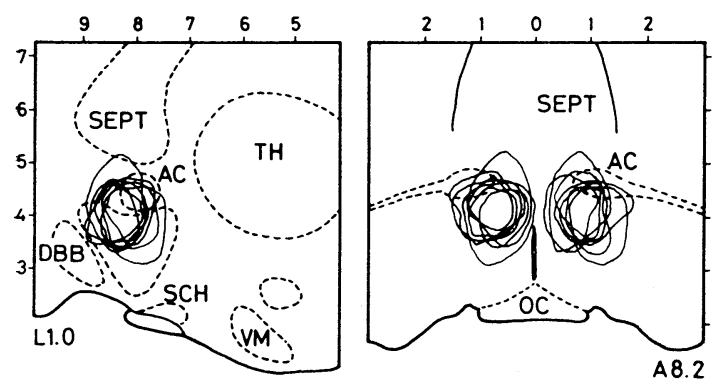

Fig. 1. Illustrations of the location of medial preoptic area lesions. Left illustration is the sagittal section $1.0 \mathrm{~mm}$ lateral from the midline. Right illustration is the frontal section $8.2 \mathrm{~mm}$ anterior to the frontal zero plane. The illustrations are modified from the atlas of Albe-Fessard et al. (1966). Abbreviations: AC, anterior commissure ; DBB, diagonal band of Broca ; OC, optic chiasm; SCH, suprachiasmatic nucleus; SEPT, septum; $\mathrm{TH}$, thalamus; VM, ventromedial nucleus.

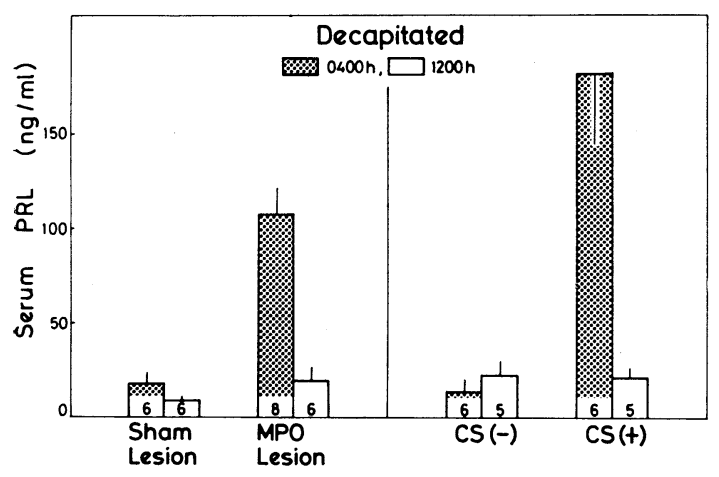

Fig. 2. Effect of medial preoptic area (MPO) lesions and cervical stimulation (CS) on PRL secretion in ovariectomized rats in which blood samples were taken by decapitation. The rats were decapitated at 0400 and $1200 \mathrm{~h}$ on the fourth day after placement of MPO lesions or CS. Each bar and its vertical line indicate the mean and S. E. M., respectively. The number of animals is given at the bottom of each bar. area lying rostral to the suprachiasmatic nucleus, and the median area located between bilateral MPO were scarcely damaged.

\section{Experiment I}

In rats in which blood samples were obtained by rapid decapitation, a marked nocturnal PRL surge was observed at $0400 \mathrm{~h}$ on the fourth day after CS or placement of bilateral MPO lesions (Fig. 2). Serum concentrations of PRL at $0400 \mathrm{~h}$ in the cervically stimulated rats and MPO-lesionsed rats rose above $100 \mathrm{ng} / \mathrm{ml}$ and were significantly greater than those at $1200 \mathrm{~h}$ $(P<0.01)$. The levels of these nocturnal PRL surges were also significantly higher than those at $0400 \mathrm{~h}$ in unstimulated rats and sham-lesioned rats $(\mathrm{P}<0.01)$.

Cervically stimulated rats bled serially from an indwelling catheter exhibited the nocturnal PRL surge, as observed in decapitated rats. Serum PRL levels in cervically stimulated rats began to increase at $2300 \mathrm{~h}$ on the third day after CS and reached peak values of $100 \mathrm{ng} / \mathrm{ml}$ at $0400 \mathrm{~h}$ on the fourth day. Thereafter the serum levels declined to the basal secretion (Fig. 3). On the other hand, the pattern of PRL secretion in MPO-lesioned rats bled from a catheter was quite different. There was no difference in the secretion pattern between MPO-lesioned rats and sham-lesioned rats. In MPO-lesioned rats, serum PRL levels remained low and showed no significant change at any time of the day $(\mathbf{P}>0.05)$.

\section{Experiment II}

In normal ovariectomized rats, MPO lesions induced a nocturnal surge of PRL secretion (Fig. 4). Serum concentrations at $0400 \mathrm{~h}$ on the fourth day in these rats was significantly greater than those at $1200 \mathrm{~h}$ $(\mathrm{P}<0.01)$. In contrast, neonatally androgenized rats did not show the nocturnal PRL surge after MPO lesions. Serum leyels 


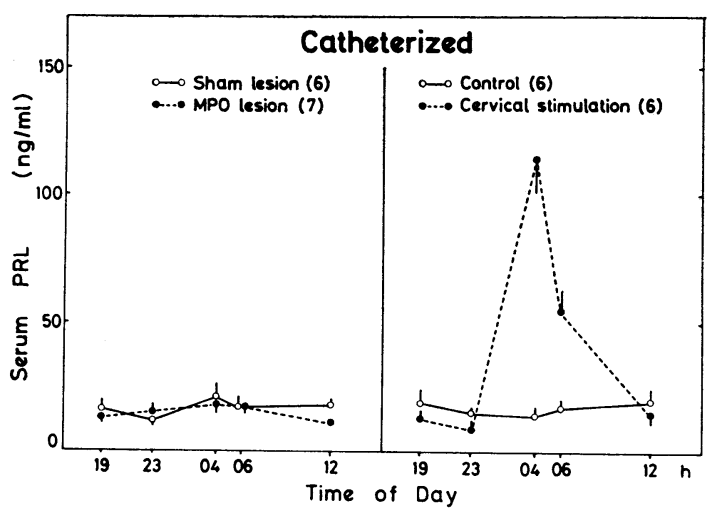

Fig. 3. Pattern of PRL secretion in MPO-lesioned rats and cervically stimulated rats in which blood samples were serially obtained through a catheter. Each point and its vertical line indicate the mean and S. E. M., respectively. The number of animals is given in parentheses.

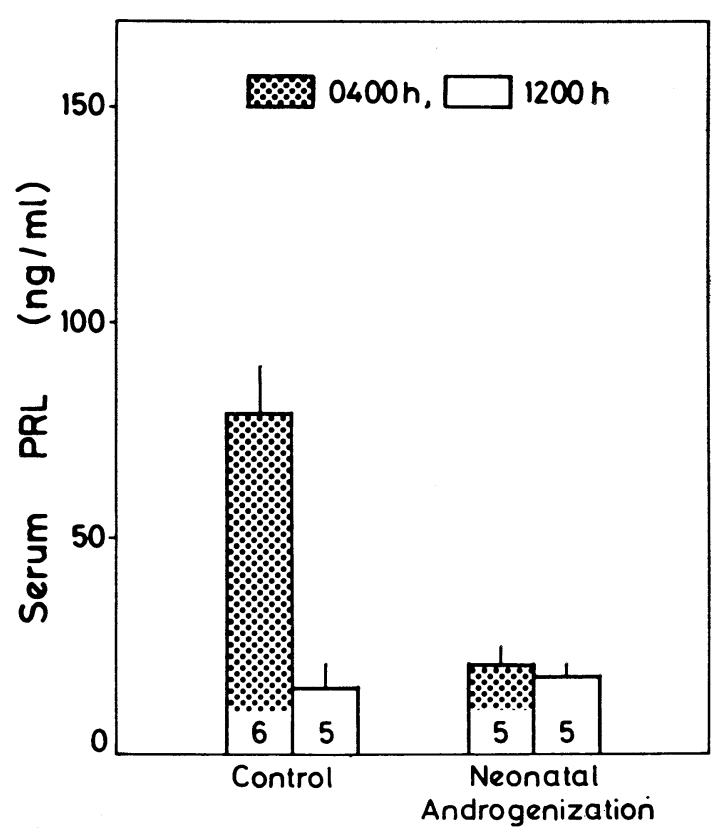

Fig. 4. Effect of MPO lesions on PRL secretion in normal female rats and rats neonatally treated with $250 \mu \mathrm{g}$ of testosterone propionate. The rats were decapitated at 0400 and $1200 \mathrm{~h}$ on the fourth day after MPO lesions. Each bar and its vertical line indicate the mean and S. E. M., respectively. The number of animals is given at the bottom of each bar. at $0400 \mathrm{~h}$ were low and did not differ from those at $1200 \mathrm{~h}$ in the neonatally androgenized rats $(P>0.05)$.

\section{Discussion}

The finding in this study that placement of MPO lesions induced a nocturnal PRL surge in ovariectomized rats without CS confirmed that of Freeman and Banks (1980). It is probable that the spontaneous occurrence of the nocturnal PRL surge at daily intervals following MPO lesions is one reason for repeated periods of pseudopregnancy in MPO-lesioned rats. There is accumulating evidence that bilateral lesions of either the suprachiasmatic nucleus (Bethea and Neill, 1980; Yogev and Terkel, 1980; Kawakami and Arita, 1981b) or the medial basal part of the suprachiasmatic area, lying immediately rostral to the suprachiasmatic nucleus, were effective in eliminating the nocturnal PRL surge induced by CS (Watanabe et al., 1980; Kawakami and Arita, 1981a). Together with these findings, the present results suggest that preoptic region contains not only the facilitatory neural mechanisms which generate the noctural PRL surge with circadian rhythms in the suprachiasmatic nucleus and the medial basal part of the suprachiasmatic area (Bethea and Neill, 1979; Pieper and Gala, 1979) but also an inhibitory system in the MPO which tonically inhibits the ultimate expression of the nocturnal PRL surge. Neural impulses caused by CS may disinhibit the inhibitory system in the MPO or activate the facilitaory surge centers in the suprachiasmatic nucleus and medial basal part of the suprachiasmatic area, or both, resulting in the initiation of the nocturnal surge.

The present study demonstrates that the nocturnal PRL surge induced by MPO lesions, but not by CS, was eliminated when the blood was collected by a catheter. It 
is probable that the elimination of the nocturnal PRL surge in MPO-lesioned rats is due to stressful stimuli attendant to catheterization and serial blood collection. This result indicates the differential regulation of the nocturnal PRL surges induced by CS and MPO lesions which seem similar to each other. Furthermore, the loss of resistance of the nocturnal PRL surge to noxious stimuli in MPO-lesioned rats suggests that neural impulses arising from stress may impinge directly on the PRL surge centers to suppress the PRL surge and that the MPO has a buffer function against the suppressing action of stress in cervically stimulated rats.

Freeman and Banks (1980) reported that MPO lesions were able to induce the nocturnal PRL surge but not diurnal surge. The disappearance of the diurnal surge in MPOlesioned rats may be explained by the possibility that the diurnal surge, which is very sensitive to the suppressing action of stress even in intact pseudopregnant rats, was suppressed by the acute effects of lesion operation in MPO-lesioned rats in which the resistance of the PRL surge to stress is lost. Furthermore, it is of interest that the spontaneous occurrence of the nocturnal PRL surge and its sensitiveness to the suppressing action of stress are observed not only in MPO-lesioned rats but also in prepubertal female rats. Kimura and Kawakami (1980) reported that immature female rats 27-28 days of age spontaneously exhibited the nocturnal and diurnal surges of PRL secretion in decapitation experiments and that catheterization attenuated the magnitude of the PRL surges in these rats. The analogy of the nocturnal PRL surge in prepubertal female rats with that in MPO-lesioned rats gives rise to the hypothesis that the neural mechanism of the MPO for the nocturnal PRL surge is not yet functional in the prepubertal stage and that the development of the neural control of the PRL surge means an establishment of the function of the MPO.

Our previous study has shown that CS did not elicit the diurnal and nocturnal PRL surges in neonatally androgenized rats (Kawakami and Arita, 1981a), suggesting that either the inability of the PRL surge centers in the suprachiasmatic nucleus and medial basal part of the suprachiasmatic area to generate the PRL surge with circadian rhythms or the inability of CS to disinhibit the inhibitory action of the MPO is responsible for the extinction of the PRL surge in neonatally androgenized rats. However, the present result that even disinhibition of the inhibitory action of the MPO by electrolytic lesion instead of CS cannot induce the nocturnal PRL surge seems to point to the former reason.

\section{Acknowledgments}

The authors thank Dr. A. F. Parlow and the Rat Pituitary Hormone Distribution Program of the NIAMDD, USA for supplying radioimmunoassay kits, and Dr. K. Wakabayashi, Gunma University, for his help and advice with radioimmunoassay. This research was supported by a Grant-in-Aid for Scientific Research from the Ministry of Education, Science and Culture, Japan.

\section{References}

Albe-Fessard, D., F. Stutinsky and S. Libouban (1966). Altas stéréotaxique du diencéphale du rat blanc. Paris, Centre National de la Recherche Scientifique.

Bethea, C. L., and J. D. Neill (1979). Prolactin secretion after cervical stimulation of rats maintained in constant dark or constant light. Endocrinology 104, 870-876.

Bethea, C. L., and J. D. Neill (1980). Lesions of the suprachiasmatic nuclei abolish the cervically stimulated prolactin surges in the rat. Endocrinology 107, 1-5.

Brown-Grant, K., M. A. F. Murray, G. Raisman and M. C. Sood (1977). Reproductive function in male and female rats following extra- and intra-hypothalamic lesious. Proc. R. Soc. Lond. Series $B$ 198, 267-278.

Clemens, J. A., E. B. Smalstig and B. D. Sawyer 
(1976). Studies on the role of the preoptic area in the control of reproductive function in the rat. Endocrinology 99, 728-735.

Freeman, M. E., M. S. Smith, S. J. Nazian and J. D. Neill (1974). Ovarian and hypothalamic control of the daily surges of prolactin secretion during pseudopregnancy in the rat. Endocrinology 94, 875-882.

Freeman, M. E., and J. A. Banks (1980). Hypothalamic sites which control the surges of prolactin secretion induced by cervical stimulation. Endocrinology 106, 668-673.

de Greef, W. J., P. van der Schoot and G. H. Zeilmaker (1980). Diurnal prolactin surges and sexual differentiation of the rat hypothalamus. Endocrinology 106, 486-489.

Kawakami, M., J. Arita and E. Yoshioka (1980). Loss of estrogen-induced daily surges of prolactin and gonadotropins by suprachiasmatic nucleus lesions in ovariectomized rats. Endocrinology 106, 1087-1092.

Kawakami, M., and J. Arita (1981a). Circadian rhythm of prolactin surges induced by cervical stimulation of the uterine cervix in the ovariectomized rat: its sexual differentiation and preoptic regulation. J. Endocrinol. 91, 325-334.

Kawakami, M., and J. Arita (1981b) Neural structures essential for the control of prolactin surges in the female rat. Exp. Brain. Res. Suppl. 3, 274-293.

Kimura, F., and M. Kawakami (1980). Two daily surges of prolactin secretion in the immature female rat. Endocrinology 107, 172-175.

Pieper, D. R., and R. R. Gala (1979). The effect of light on the prolactin surges of pseudopregnant and ovariectomized, estrogenized rats. Biol. Reprod. $20,727-732$.

Smith, M. S., and J. D. Neill (1976). A "critical period" for cervically-stimulated prolactin release. Endocrinology 98, 324-328.

Watanabe, H., H. Furuya, R. Okada, K. Yamanouchi and Y. Arai (1980). Interruption, of pseudopregnancy by rostral periventricular preoptic lesions in the female rat. Endokrinologie 76, 181184.

Wiegand, S. J., E. Terasawa, W. E. Bridson and R. W. Goy (1980). Effects of discrete lesions of preoptic and suprachiasmatic structures in the female rat. Neuroendocrinology 31, 147-157.

Yogev. L., and J. Terkel (1980). Effects of photoperiod, absence of photic cues, and suprachiasmatic nucleus lesions on nocturnal prolactin surges in pregnant and pseudopregnant rats. Neuroendocrinology 31, 26-33.

Zeilmaker, G. H. (1963). Experimental studies on the regulation of corpus luteum function in castrated male rats bearing a transplanted ovary. Acta Endocrinol 43, 246-254.

Zeilmaker, G. H. (1964). Aspects of the regulation of corpus luteum function in androgen-sterilized female rats. Acta Endocrinol. 46, 571-579. 